\title{
Community exoplanet imaging data challenge for Roman CGI and starshade rendezvous
}

\author{
Margaret C. Turnbull $\odot,{ }^{\text {a,* }}$ Neil Zimmerman $\odot,{ }^{b}$ Julien H. Girard $\odot,{ }^{\text {c }}$ \\ Sergi R. Hildebrandt $\odot$, ${ }^{\text {d,e }}$ Zhexing Li $\odot,{ }^{\mathrm{f}}$ Ell Bogat $\odot,{ }^{\mathrm{b}}$ \\ Junellie Gonzalez-Quiles $\odot$, ${ }^{\text {g }}$ Christopher Stark, ${ }^{\mathrm{b}}$ Avi Mandell, ${ }^{\mathrm{b}}$ \\ Tiffany Meshkat $\odot$, and Stephen R. Kane ${ }^{\mathrm{f}}$

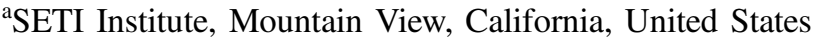 \\ bNASA Goddard Space Flight Center, Greenbelt, Maryland, United States \\ ${ }^{\mathrm{c}}$ Space Telescope Science Institute, Baltimore, Maryland, United States \\ d Jet Propulsion Laboratory, California Institute of Technology, Pasadena, California, United States \\ ${ }^{\mathrm{e} C}$ California Institute of Technology, Division of Physics, Mathematics, and Astronomy, \\ Pasadena, California, United States \\ ${ }^{\mathrm{f}}$ University of California, Department of Earth and Planetary Sciences, Riverside, \\ California, United States \\ g Johns Hopkins University, Department of Earth and Planetary Sciences, Baltimore, \\ Maryland, United States \\ hIPAC, California Institute of Technology, Pasadena, California, United States
}

\begin{abstract}
Operating in an unprecedented contrast regime $\left(10^{-7}\right.$ to $\left.10^{-9}\right)$, the Roman Coronagraph Instrument (CGI) will serve as a pathfinder for key technologies needed for future Earth-finding missions such as HabEx and LUVOIR. The Roman Exoplanet Imaging Data Challenge (Roman EIDC) was a community engagement effort that tasked participants with extracting exoplanets and their orbits for a 47-UMa-like target star given: (1) 15 years of simulated precursor radial velocity (RV) data and (2) six epochs of simulated imaging taken over the course of the Roman mission. Led by the Turnbull CGI Science Investigation Team, the Roman EIDC was preceded by four tutorial "hack-a-thon" events in Baltimore, Pasadena, New York City, and Tokyo. The Roman EIDC officially launched in October 2019 and ran for 8 months, offering a unique opportunity for exoplanet scientists of all experience levels to get acquainted with realistic near-future imaging data. The Roman EIDC simulated images include four epochs with CGI's Hybrid Lyot Coronagraph (HLC) plus two epochs with a starshade (SS) assumed to arrive as part of a Starshade Rendezvous later in the mission. We focus on our in-house analysis of the outermost planet "d," for which the SS's higher throughput and lower noise floor present a factor of $\sim 4$ improvement in the signal-to-noise ratio over the narrow-field HLC. We find that, although the RV detection was marginal for planet d, the precursor RV data enabled the mass and orbit to be constrained with only two epochs of SS imaging. Including the HLC images in the analysis results in improved measurements over RV + SS alone, with the greatest gains resulting from images taken at epochs near maximum elongation. Combining the two epochs of SS imaging with the RV + HLC data resulted in a factor of $\sim 2$ better orbit and mass determinations over RV + HLC alone. In summary, the Roman CGI, combined with precursor RV data and latermission SS imaging, forms a powerful trifecta in detecting exoplanets and determining their masses, albedos, and system configurations. While the Roman CGI will break new scientific and technological ground with direct imaging of giant exoplanets within $~ 5 \mathrm{AU}$ of $V \tilde{5}$ and brighter stars, a Roman Starshade Rendezvous mission would additionally enable the detection of planets out to $\sim 8 \mathrm{AU}$ in those systems. (C) 2021 Society of Photo-Optical Instrumentation Engineers (SPIE) [DOI: 10.1117/1.JATIS.7.2.021218]
\end{abstract}

Keywords: Roman space telescope; coronagraph; starshade; high contrast imaging; exoplanet; data challenge; HabEx.

Paper 20175SS received Dec. 1, 2020; accepted for publication May 12, 2021; published online May 28, 2021.

*Address all correspondence to Margaret C. Turnbull, turnbull.maggie@gmail.com 


\section{Introduction}

\subsection{Roman Mission and Exoplanet Coronagraph}

The 2.4-m Nancy Grace Roman Space Telescope (Roman; formerly the Wide Field Infrared Survey Telescope, WFIRST) ${ }^{1}$ is a NASA mission set to launch in the mid-2020s and operate at the second Sun-Earth Lagrange point (SEL2). Roman will carry two scientific instruments: (1) the Wide Field Instrument, providing imaging resolution comparable to that achieved by the Hubble Space Telescope but with 100 times the field of view and (2) a high-contrast, small field of view Coronagraph Instrument (CGI). The Roman CGI is intended to serve as a pathfinder instrument for future exoplanet imaging missions such as $\mathrm{HabEx}^{2}$ by demonstrating key starlight suppression technologies, including the first active wavefront control in space. ${ }^{3}$ CGI aims to achieve detection limits of $10^{-7}$ (minimum requirement) to $10^{-8}$ (current best estimate) at separations of $\sim 0.15$ to 1.5 arcsec from bright $(V<6)$ host stars, which would enable imaging and low-resolution spectroscopy of giant planets in reflected starlight. ${ }^{4}$ The simulations in the Roman Exoplanet Imaging Data Challenge (Roman EIDC) employed the most recent end-to-end observing scenario models (OS6),$^{5}$ which enabled detections down to $\sim 10^{-9}$. As of this writing, the Roman mission has passed its preliminary design review and entered phase $\mathrm{C}$ (final design and fabrication).

\subsection{Goals and Scope of the Data Challenge}

The goals of the Roman EIDC $^{6}$ are as follows:

- Familiarize the exoplanet community with the CGI data products and with working in a new contrast regime that enables the detection of mature giant planets in reflected starlight.

- Develop, use, and improve data simulation and analysis tools, including combining RV and imaging data and using a variety of postprocessing and PSF subtraction techniques.

- Explore the detection space enabled or enhanced by combining precursor RV and latemission starshade (SS) observations with the CGI data.

- And train and foster collaborations between future leaders of exoplanet science.

The scope of the Roman EIDC was to unveil an exoplanetary system hidden in realistic CGI data, which includes high fidelity wavefront control residuals, a realistic EMCCD detector model, and astrophysical contamination. Participating teams were given six simulated imaging epochs for a nearby star (modeled after $47 \mathrm{UMa}$ ), calibration files corresponding to OS6, 15 years of simulated precursor radial velocity (RV) data (described in Sec. 2.3), and basic stellar system information (V-band magnitude, distance, mass, spectral type, and proper motion). Participants were not told the number of planets present nor any prior information about background stars, galaxies, or exozodiacal light. From these data, participants were asked to determine the number of planets detected and to find their orbital parameters, masses, radii, and albedos. Our three-planet 47 UMaanalog system was designed so that the planets (b, c, and d) would be in stable orbits and fall in or out of the field of view at various detection levels in the various epochs. In only one instance was the entire planetary system (marginally) detectable in a single epoch. The imaging and RV simulations will be discussed in detail in a future publication. ${ }^{7}$

\subsection{Challenge in Four Steps}

The Roman EIDC was rolled out in four increasingly difficult steps to help participants work in a logical manner and avoid overwhelming the teams. Participants submitted the results of each step to receive the next package of simulated data, as follows:

Step 1: HLC only. Identify all point sources in four HLC epochs, disentangle planets from any background sources, and provide system census and astrometry.

Step 2: HLC $+R V$ priors. Compute orbital parameters and masses using the above four HLC epochs plus priors from RV data. 
Step 3: $H L C+R V+S S$. Refine the orbital parameters and masses of all detected planets using two additional SS epochs.

Step 4: For each planet, measure the phase curve (assuming a Lambertian reflectance function) and derive the radius and albedo from a provided mass-radius relationship.

Importantly, the SS images were not provided until step 3 because these data would have undermined the "blind" analyses of the prior epochs by clearly revealing planets that are only marginally detected with the HLC. Figures 1 and 3 show the science grade data products (postPSF subtraction) for the HLC and SS, respectively.

\subsection{Hack-a-Thons and a Legacy Tutorial Suite}

To engage the community prior to launching the Roman EIDC, we held four tutorial "hack-athon" events in Baltimore, Los Angeles, New York, and Tokyo. Hack-a-thon participants were given two rehearsal data sets as well as a suite of Jupyter notebooks (mainly written in Python) to begin working with the data. This training material was developed and improved over the duration of the challenge, and it is now available for public use. ${ }^{8}$ Altogether, a diverse group of over 70 people attended the hack-a-thons, a subset of whom ultimately participated in the full data challenge either individually or in teams. In an upcoming paper, ${ }^{9}$ we will report on the Roman EIDC results in detail.

\section{Data Simulations}

\subsection{HLC Images}

The baseline imaging mode of CGI is a Hybrid Lyot Coronagraph (HLC) operating in a bandpass centered at $575 \mathrm{~nm}$ (the 546 to $604 \mathrm{~nm}$ "band 1" filter of CGI). This configuration provides the smallest inner working angle (IWA) of the available coronagraph modes, 150 mas, which results in the best overall sensitivity for detecting exoplanets in reflected starlight. The data challenge files include four epochs of simulated HLC images of the scientific target at time intervals $\Delta T=0.0,0.15,1.0$, and 2.0 years from an initial observation on November 1, 2026. The timevarying residual starlight pattern, including speckles and pointing jitter effects, is based on the "Observing Scenario 6" (OS6) PSF time series prepared by the CGI project's integrated modeling team in $2018 .^{5}$ The OS6 PSF time series includes alternating observatory roll angles to assist in discriminating astrophysical signals from quasistatic speckles. Therefore, the simulated HLC images are provided as co-added images with a total integration time of $66,000 \mathrm{~s}(\sim 18 \mathrm{~h})$ in each of two roll angles $26 \mathrm{deg}$ apart, along with the science target observation. None of the
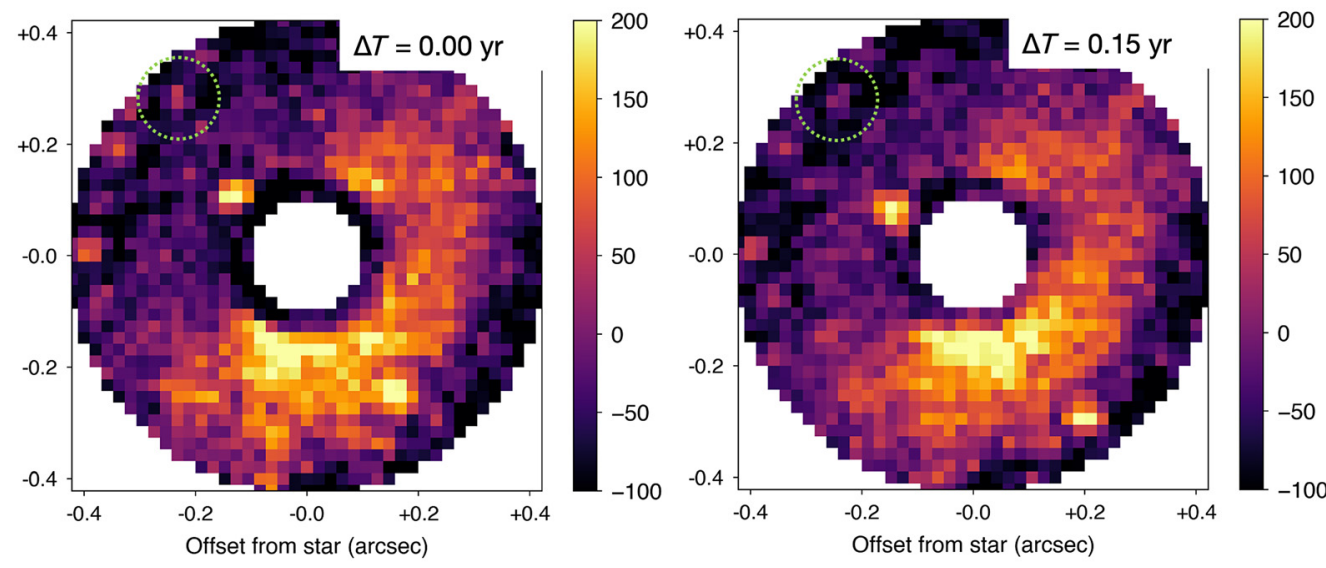

Fig. 1 Simulated CGI HLC data after applying RDI and roll combination to the co-added images at two epochs, displayed in units of integrated photoelectrons. The faint source corresponding to planet "d" is circled in green. The data analysis presented in this paper was carried out "in house" by the Roman CGI Turnbull Science Investigation Team members. 
provided images have been postprocessed, and it was left to the participants to apply either reference differential imaging (RDI) or angular differential imaging to partly subtract the residual starlight pattern.

The result of our "in-house" image reduction applied to two simulated HLC data epochs is shown in Fig. 1. There are two relatively bright point sources: one is a planet (NE quadrant, near the occulting mask) and the other is a background star (SW quadrant, near the edge of the field). In addition, the reduced images reveal diffuse scattered light from a debris disk with a peak surface brightness of 19.5 mag arcsec${ }^{-2}$ in $V$-band (equivalent to 10 "zodi" surface brightness units). Lastly, the source that we highlight for this article is a faint outer planet, circled in green in Fig. 1. This planet "d" source would be indistinguishable from speckle noise if these HLC images were the only evidence for its existence. However, planet $d$ reappears as a much higher signal-to-noise ratio (SNR) point source in the later SS rendezvous images (Sec. 2.2), with motion consistent with a Keplerian orbit and physically consistent with a weak, long-period residual feature in the data challenge RVs (Sec. 2.3).

\subsection{Starshade Images}

The SS images were generated with SISTER, ${ }^{10}$ an open-source software capable of performing SS simulations with high optical fidelity. We chose the 425- to 552-nm band of the Starshade Rendezvous Probe (SRP) ${ }^{11}$ see Fig. 2, because it is the closest channel to the HLC simulated images. The simulation uses nominal instrument parameters consistent with Roman end-of-life (EOL) operation and includes epochs consistent with the required solar exclusion angles of the mission.

\subsubsection{Telescope and detector}

The telescope pupil includes the secondary mirror central obscuration and struts and is $2.36 \mathrm{~m}$ in diameter. The detector noise follows the expected Roman EMCCD model at its EOL. The particular values of the different parameters come from the Roman Space Telescope parameters listed online. ${ }^{12}$ The readout noise is $100 \mathrm{e}^{-}$perframe, EM gain is 1000 , dark current at EOL is $0.77 \mathrm{e}^{-} / \mathrm{pix} / \mathrm{h}$, and clock-induced charge noise is $0.02 \mathrm{e}^{-} / \mathrm{pix} /$ frame. To compare the contribution of SS data with the CGI data more easily, the total integration time set for both epochs with SS data was 1.5 days, the same as for the CGI HLC epochs. The detector quantum efficiency $(\mathrm{QE})$ is based on laboratory measurements of Roman's EMCCD detector. The effective QE across the 425 to $552 \mathrm{~nm}$ is 0.45 , and it is the result of the actual QE and further losses due to cosmic rays, charge transfer efficiency, and hot pixels. The pixel scale in the Roman coronagraph instrument is $21.85 \mathrm{mas} / \mathrm{pixel}$. We have assumed that all of the pixels are identical without any hot pixels. The simulation does not include contamination from cosmic rays or sources of noise not intrinsic to the detector electronics. The end-to-end optical throughput

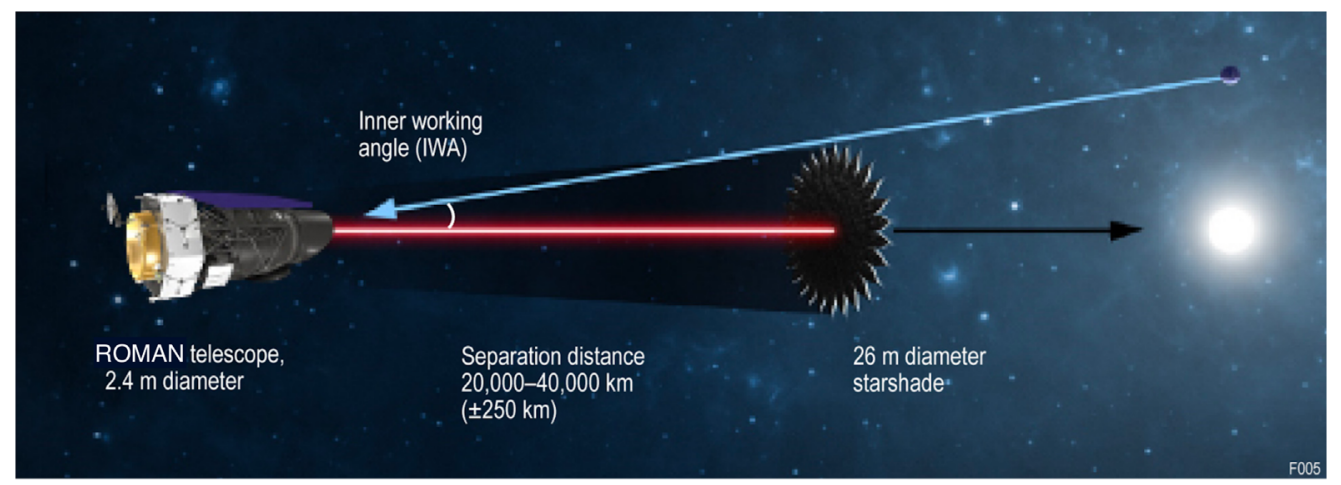

Fig. 2 The geometry of the SS and Roman telescope in the SRP mission concept. ${ }^{11}$ The SS's IWA is the apparent size of the SS's radius as seen by the Roman telescope. In the 425 to $552 \mathrm{~nm}$ passband, the IWA is 72 mas. 
is the product of reflection losses in the telescope $(0.81)$, the coronagraph instrument $(0.60$, exclusive of coronagraph masks which are not used), and the SS dichroic filter (0.9) for a net throughput of 0.44 .

\subsubsection{Starshade}

The SS has 24 petals and is $26 \mathrm{~m}$ in diameter. The SS's geometric IWA is the apparent size of the SS's radius as seen by the Roman telescope and in the 425- to 552-nm passband is 72 mas, as shown in Fig. 2. For sources located far from the SS's IWA, the optical response (PSF FWHM = 40.6 mas at mid-band) is that of the Roman telescope, including the secondary mirror and six supporting struts.

\subsubsection{Starshade imperfections}

In this paper, we define "contrast" as the ratio of the intensity averaged over an image plane resolution element (typically the PSF FWHM) to the peak intensity of the target star when the SS is not present. The current best estimate of the SS contrast in the SRP report ${ }^{11}$ is $4 \times 10^{-11}$. In the Roman EIDC, we considered an imperfect SS that would allow us to simulate possible manufacturing and deployment errors of the SS. The SRP report sets the error budget of the SS contrast to $1.0 \times 10^{-10}$. However, the locations of the planets on the image plane fall well outside the 72-mas IWA for all of them (the closest angular separation being 116 mas). At those angular distances, the contrast of the SS improves substantially. To explore any potential effect of the residual starlight on the planets' analysis, we artificially degraded the performance of the SS to an average contrast over the passband of $1.4 \times 10^{-10}$ at the IWA. This degraded contrast performance was achieved by displacing the petals in-plane as 24 independent rigid bodies with radial and azimuthal offsets such that the root-mean-square displacement of all points along the petals was $0.56 \mathrm{~mm}$. We did not include shear displacement of the SS relative to the line of sight between the telescope and target star (expected to be $<2$ mas) nor any tilt of the SS plane with respect to the telescope. Both of these effects have negligible impact on the exoplanet analyses. ${ }^{10}$

\subsubsection{Solar glint}

For both SS imaging epochs, the sun is located $60 \mathrm{deg}$ from the SS normal, which is a median value among planned observations and is oriented at $0 \mathrm{deg}$ from horizontal. Solar glint (sunlight reflected off of the SS into the telescope) is computed under a "worst case scenario" assumption that the SS edges are not coated with any antireflection coating. The resulting solar glint intensity is therefore $\sim 10$ times greater than current best estimates to better explore its impact on the data analysis.

\subsubsection{Astrophysical components}

The astrophysical components of the SS simulations include the three-planet system itself, plus residual starlight from an imperfect SS, solar glint due to sun's light scattered through the SS petals, local zodiacal light, exozodiacal dust light, and a background galaxy. The planets' apparent separations for epoch $\Delta T=3$ years are 116.6, 271.0, and 488.2 mas, respectively, and for epoch $\Delta T=4$ years they are $159.6,182.7$, and 502.1 mas. In all cases, the projected distance is significantly larger than the geometric IWA of the SS in this configuration (72 mas).

We assumed a Lambertian phase function to derive the intensity of the reflected light from the planets, which is good enough to fulfill the aims of the data challenge. The flux ratios for epoch $\Delta T=3$ years are $5 \times 10^{-10}, 5.6 \times 10^{-10}$, and $6.7 \times 10^{-10}$, for planets b, c, and d, respectively, and for epoch $\Delta T=4$ years they are $2.4 \times 10^{-9}, 1.2 \times 10^{-10}$, and $4.7 \times 10^{-10}$, respectively. For comparison, Earth's flux ratio at quadrature is $1.1 \times 10^{-10}$ at $500 \mathrm{~nm}$. The planet orbital configuration was chosen to provide low flux ratios during the SS epochs to better demonstrate the capability of SS imaging. 

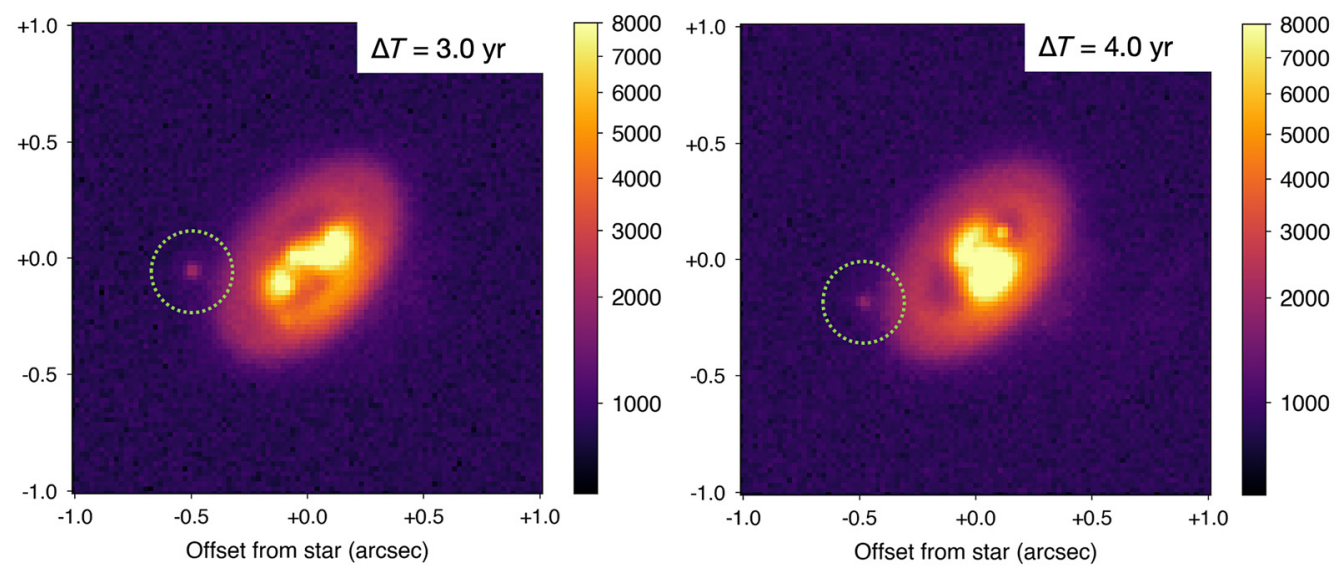

Fig. 3 Co-added Roman CGI/SS images of the data challenge target, without any postprocessing, displayed in units of integrated photoelectrons. The point source corresponding to planet "d" is circled in green.

The exozodiacal cloud is assumed to have a density five times that of the solar system's zodiacal cloud. Its dust distribution was derived from an N-body dynamical simulation of dust particles under the gravitational influence of a co-orbiting planet. ${ }^{13}$ The dust grains in this model tend to become trapped in mean motion resonances with a planet. In the data challenge images, the scattered light from these resonant dust structures produces diffuse intensity enhancements that co-orbit with the innermost planet "b." For the extragalactic background object, we used the Haystacks data cubes ${ }^{14}$ and selected the brightest background galaxy from the HST deep field survey. In Sec. 3, we provide the relative contribution of each component for the case of planet $d$.

Simulated images for the two epochs with the SS without any postprocessing are shown in Fig. 3. Both images show planet d clearly inside the dotted circle. In epoch $\Delta T=3$ years, planet $\mathrm{c}$ is located near $(-0.10,-0.25)$ arcsec and can be seen as a faint perturbation to the exozodiacal dust, while planet $\mathrm{b}$ is masked by a bright clump of the exozodiacal light. In epoch $\Delta T=4$ years, planet b is clearly visible near $(0.10,0.15)$ arcsec, while planet c is masked by the exozodiacal light. The images also show how the resonant structure of the exozodiacal revolves around $47 \mathrm{UMa}$ from one epoch to the following one and its impact on planet detection.

\subsection{Precursor Radial Velocity Data}

Synthetic RV data points were created spanning 15 years: first 10 years with RV instrumental precision of $1 \mathrm{~m} / \mathrm{s}$ similar to instruments such as Keck HIRES and an additional 5 years with $0.3 \mathrm{~m} / \mathrm{s}$ taking into account the emerging extreme precision RV instruments such as the WIYN NEID spectrograph. A total of 200 data points were created by solving Kepler's equation and superposing individual signals from each planet. The number of data points and the length of coverage is similar to the archival RV coverage for $47 \mathrm{UMa}$, except that the data points are spread out nearly evenly (i.e., in the most optimal way). No planet-planet interactions are included in the RV data, since such effects are not observable with RV.

To mimic different sources of noise, each RV data point was passed through a Gaussian filter to randomize the velocity measurements, with the sigma calculated from the quadrature sum of instrumental error and stellar jitter. The jitter value of around $2.1 \mathrm{~m} / \mathrm{s}$ was estimated using the method from Isaacson and Fischer ${ }^{15}$ assuming the stellar mass and $S$-index values of a $47 \mathrm{UMa}$ host star. The total RV as well as the individual phase-folded RV for each planet is shown below. The figure is generated using RV modeling toolkit $\mathrm{RadVel}{ }^{16}$ with all of the parameters fixed so that the outermost planet can be properly displayed. Parameters for each planet such as the orbital period $(\mathrm{P})$, semiamplitude $(\mathrm{K})$, and eccentricity (e) are shown in the upper right corner of each phase-folded panel. 


\section{Revealing Planet d with the Starshade}

We designed the fictitious data challenge planetary system to probe a range of detection difficulties. The outermost planet "d" was chosen to represent a particularly stressing case for CGI's sensitivity limits: a roughly Saturn-sized planet at a 7.7 AU semimajor axis, with a visible wavelength geometric albedo of 0.5 . At a resulting planet-to-star flux ratio of $\sim 10^{-9}$, the intensity of planet $d$ is almost equal to the residual speckle noise standard deviation in the HLC image after classical RDI and roll combination. Therefore, in the context of HLC data alone, planet d does not present an obvious source candidate among the various residual subtraction features. However, when analyzed in concert with the SS images and supporting RVs, a more compelling picture emerges.

Owing to the higher throughput and much lower systematic noise of the Roman/SS observing configuration, the SNR of the planet d point source increases from SNR $\sim 1$ to SNR $\sim 4$ in the SS imaging epochs ( $\Delta T=3$ years and $\Delta T=44$ years). Due to its projected location for outside the IWA on the image plane (488.2 mas in $\Delta T=3$ years and 502.1 mas in $\Delta T=4$ years compared with the 72-mas IWA), the contributions from the residual starlight and solar glint to planet d's signal are negligible compared with other background sources. Within a circular area with a diameter equivalent to 1 FWHM, the background source contribution is $\sim 30 \%$ of planet d's signal. Furthermore, $94 \%$ of the background source is due to local zodiacal light and $6 \%$ to the exozodiacal cloud light. The spatial distribution of the local zodiacal light is very smooth at the angular scales of these images, and it can easily be subtracted, leaving only the shot noise from the exozodi signal. As mentioned before, the overall SNR of planet $d$ is $\sim 4$.

These two SS point source detections (one in each SS epoch), considered in combination with the physically consistent long-period residual sinusoid in the RVs (Fig. 4), would strongly motivate a retrospective search for a planet d signal in the earlier HLC data. Scenarios like this one, where a new detection is reinforced by the recovery of a previously missed signal in older data, are common in observational astronomy. One famous example in high-contrast imaging is the detection of the HR 8799 planets b, c, and d in archival Hubble Space Telescope data, in NICMOS images acquired 10 years before the discovery of those objects. ${ }^{17}$

The regions containing the planet d signal in the first two HLC images are circled in Fig. 1. To carry out our "in-house" astrometry analysis, we measured the point source centroids by cross-correlating a PSF model with a small cutout centered on the signal region. Our astrometry data points are plotted as the filled-in circles in Fig. 5, alongside different symbols representing the astrometry reported by data challenge participants, whose results will be described in a forthcoming publication. Note that our in-house analysis could not recover even a marginal signal in the third epoch HLC image. Furthermore, since planet d passes outside the HLC outer working angle after this time, no detection is possible in the fourth imaging epoch $(\Delta T=2$ years).

\subsection{Orbit Fitting}

By combining the two epochs of SS astrometry (Fig. 3) with the residual sinusoidal feature in the RVs [Fig. 4(c)], it is possible to place some preliminary constraints on the Keplerian orbit. In our orbit fitting trials with the open-source RadVel ${ }^{16}$ and orbitize! ${ }^{18}$ packages, we found that, when applying priors on period, eccentricity, and ascending node position angle with the two epochs of SS astrometry, orbitize! constrains the semimajor axis to a $68 \%$ confidence interval of 7.4 to 10.5 $\mathrm{AU}$ and the inclination angle to a $68 \%$ confidence interval of 41 to $56 \mathrm{deg}$.

With the addition of the weaker point source detections in the HLC images, the fit is improved by the increased time baseline. In Table 1, we list the $68 \%$ confidence intervals of the inclination angle and planet mass retrieved from the combined orbit fit, alongside the true values used to generate the simulated data. The planet mass constraints are modest, being limited by the quality of the RV signal and the fact that the imaging astrometry only covers one-fifth of planet d's 21-year orbital period. Even with all four epochs of astrometry, the confidence interval spans more than a factor of 2 in mass, from 0.24 Jupiter masses $\left(M_{\text {Jup }}\right)$ to $0.55 M_{\text {Jup }}$. The other two data challenge planets are situated at smaller orbital radii, with semimajor axes 2.3 AU and 4.2 AU. In those cases, the narrower confidence intervals of the RV semiamplitudes, along with larger orbital period coverage of the imaging data, reduce the final mass uncertainties to within 

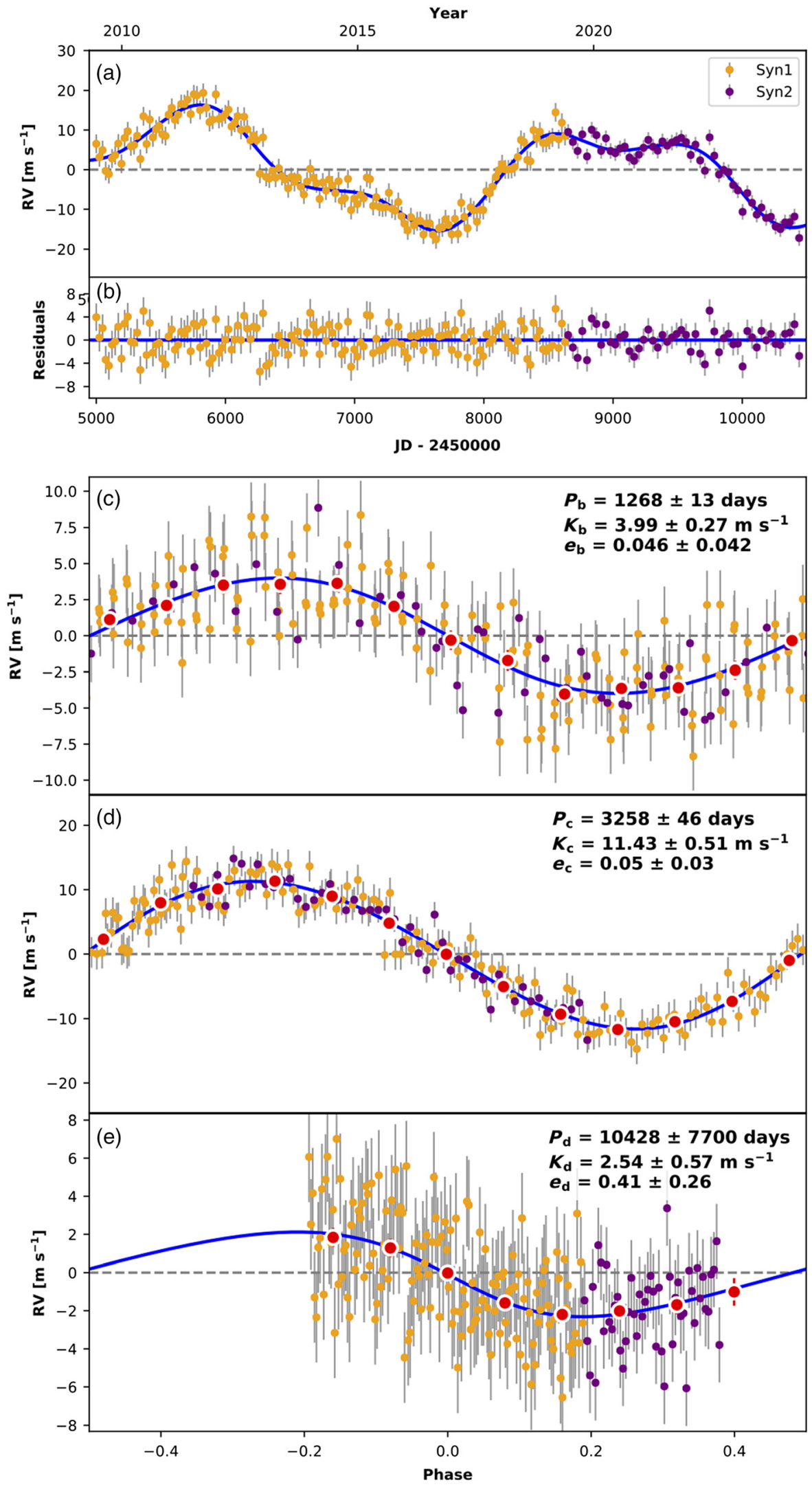

Fig. 4 Synthetic RV for the system. (a), (b) The total RV with the best fit three-planet model along with residuals. (c), (d), and (e) Individual phase-folded RVs for each planet. 


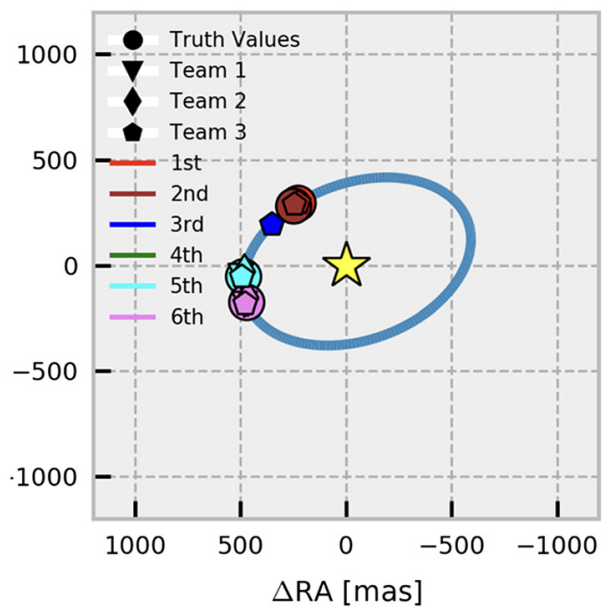

Fig. 5 Astrometry of planet $d$ reported by data challenge participants. The true projected Keplerian orbit is shown by the blue ellipse. The first two epochs are very low (SNR 1) HLC detections that would most likely only be recovered based on retrospective analysis after eventual Roman Starshade Rendezvous detections.

Table 1 Estimated orbital inclination and mass of planet $d$, based on combining RV priors with various subsets of astrometry. These measurements are from an "in-house" analysis of the simulated data; results from the data challenge participants are deferred to forthcoming publications.

\begin{tabular}{lcccc}
\hline \hline & $\begin{array}{c}\text { Two epochs } \\
\text { (HLC only) }\end{array}$ & $\begin{array}{c}\text { Two epochs } \\
\text { (SS only) }\end{array}$ & $\begin{array}{c}\text { Four epochs } \\
\text { (HLC and SS) }\end{array}$ & $\begin{array}{r}\text { True } \\
\text { value }\end{array}$ \\
\hline Inclination (deg) & $71_{-28}^{+38}$ & $50_{-9}^{+6}$ & $48_{-6}^{+5}$ & 48 \\
Mass $\left(M_{\text {Jup }}\right)$ & $0.25_{-0.10}^{+0.33}$ & $0.34_{-0.12}^{+0.24}$ & $0.35_{-0.11}^{+0.20}$ & 0.32 \\
\hline \hline
\end{tabular}

$20 \%$ of their true values. We will describe the results for these other data challenge planets, with emphasis on the participants' analysis, in forthcoming publications. Because those inner planets are brighter and have shorter orbital periods, the HLS data are sufficient for detecting and estimating their complete orbital parameters to similar precision, without SS observations.

\subsection{Photometry and Phase Curve Analysis}

One of the aspects of future reflected starlight direct imaging will be the ability to observe the photometric variation over the course of the orbit due to the changing star-planet-observer phase angle. Combined knowledge of the orbit, along with an inferred planet radius, will enable observers to estimate the bulk geometric albedo from a flux ratio time series. As a demonstration, we used our imaging photometry of planet $d$, in combination with the previous orbit fit, to estimate the planet's geometric albedo, assuming a Lambertian sphere scatterer.

Before fitting for the planet's albedo, we first used the posterior mass estimate from the orbit fit and RV semiamplitude and then inferred the planet's radius from an empirical mass-radius relationship along with the approximate equilibrium temperature of planet d. ${ }^{19}$ A simple leastsquares optimization finds the albedo that minimizes the distance between the four epochs of photometry and the Lambertian phase curve associated with the orbit posteriors. The result, in Fig. 6, illustrates the four photometric data points, the best-fit flux curve, and the flux curve of the true planet parameters.

One of the dramatic features shown in Fig. 6 is the improved photometric constraints enabled by the higher SNR SS detections. A slight bias in the SS photometry causes the albedo to be underestimated $(0.3$ versus the true value of 0.5$)$, but this bias is still much smaller than the overall uncertainty of the albedo estimate. The fit shown in Fig. 6 corresponds only to the best 


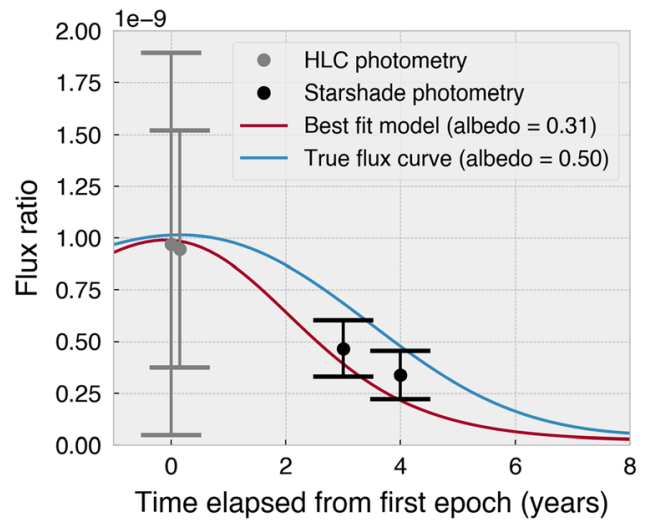

Fig. 6 Best fit (red) to the geometric albedo of planet d using photometry and orbit posteriors, assuming a Lambertian sphere scatterer. The true flux curve is shown in blue.

estimated orbit parameters and mass, not the envelope of all photometric phase curves within the confidence intervals of all orbital elements. If we repeat the fit at the minimum and maximum extremes of the $68 \%$ confidence intervals of all orbit parameters derived from the data, then the albedo confidence interval spans 0.04 to 0.73 . A longer time series with photometry at the SS precision would enable better constraints.

\section{Summary}

The Roman CGI, combined with precursor RV data and late-mission SS imaging, forms a powerful trifecta in detecting exoplanets and determining their masses, albedos, and system configurations. While the CGI is nimbler than an SS and can more readily target specific epochs to reduce uncertainty in planet orbits, the higher throughput of the SS enables detection and study of planets much farther out in the system and resonant exozodiacal dust structures. In the Roman CGI Exoplanet Data Challenge, we found that even a marginal prior RV detection of outermost planet $d$ enabled its mass and orbit to be constrained with only two epochs of SS imaging. Including the HLC images in the analysis results in improved measurements over RV + SS alone, with the greatest gains resulting from images taken at epochs near maximum elongation. Combining the two epochs of SS imaging with the RV + HLC data resulted in a factor of $\sim 2$ better orbit and mass determinations over RV + HLC alone. While the Roman CGI is expected to break new ground with direct detection of giant exoplanets within $\sim 5$ AU of $V \sim$ 5 and brighter stars, a Roman Starshade Rendezvous mission would additionally enable the detection and characterization of planets out to $\sim 8 \mathrm{AU}$ in those systems.

The Roman CGI Exoplanet Data Challenge has proven to be an excellent way to (1) engage the community in working with the intricacies of the first mission to perform wavefront control in space and (2) motivate the community to invest in larger missions that will enable the study of mature exoplanet systems, including habitable planets, in reflected starlight. This effort also generated many interactions between open-source package developers (e.g., orbitize! and RadVel) and a diverse group of exoplanet scientists running them, resulting in (1) key enhancements to those packages and (2) the creation of a legacy tutorial suite that is now available online for future training exercises.

\section{Acknowledgments}

This work was funded by NASA Grant NNG16PJ27C, which supports the Turnbull Roman CGI Science Investigation Team. We thank the Hayden Planetarium and Jackie Faherty for an excellent tour of known exoplanet host stars and the Flatiron Institute for hosting the New York City hack-a-thon event. We thank STScI, IPAC, and Motohide Tamura and Masayuki Kuzuhara (University of Tokyo and Astrobiology Center) for hosting hack-a-thons in Baltimore, Pasadena, and Tokyo. We thank John Krist and the JPL project science team for the OS6 
simulations, and we thank Sarah Blunt for her many interactions with the Roman EIDC team and participants and for her ongoing work enhancing the extremely useful orbitize! package. We also thank BJ Fulton for contributing his expertise with RadVel. Finally, we thank all of the Hack-athon and Data Challenge participants for joining us in this community venture and providing extensive feedback over the last two years.

\section{References}

1. R. Akeson et al., "The wide field infrared survey telescope: 100 Hubbles for the 2020s," arXiv:1902.05569 (2019).

2. B. S. Gaudi et al., "The Habitable Exoplanet Observatory (HabEx) 284 mission concept study final report," arXiv:2001.06683 (2020).

3. V. Bailey et al., "Key technologies for the wide field infrared survey telescope coronagraph instrument," arXiv:1901.04050 (2019).

4. B. Mennesson et al., "Paving the way to future missions: the Roman space telescope coronagraph technology demonstration," arXiv:2008.05624 (2020).

5. J. Krist, B. Nemati, and B. Mennesson, "Numerical modeling of the proposed WFIRSTAFTA coronagraphs and their predicted performances," J. Astron. Telesc. Instrum. Syst. 2, 011003 (2016).

6. J. Girard et al., "Roman Exoplanet Imaging Data Challenge (Roman EIDC)," www .exoplanetdatachallenge.com.

7. N. Zimmerman et al., "Roman Community Exoplanet Imaging Data Challenge: I. Design and Simulations," in prep (2021).

8. J. Girard et al., "Roman exoplanet imaging data challenge tutorials," https://github.com/ wfirst-cgi/Roman-CGI-Data-Challenge-Tutorials.

9. J. Girard et al., "Roman Exoplanet Imaging Data Challenge: II. Implementation, Results and Lessons Learned," in preperation (2021).

10. S. R. Hildebrandt et al., "SISTER: Starshade Imaging Simulation Toolkit for Exoplanet Reconnaissance," J. Astron. Telesc. Instrum. Syst. 7(2), 021217 (2021).

11. S. Seager et al., "Starshade rendezvous probe study report," 2019, https://smd-prod.s3 .amazonaws.com/science-red/s3fs-public/atoms/files/Starshade2.pdf.

12. IPAC, "Roman Space Telescope parameters," https://wfirst.ipac.caltech.edu/sims/Param_db .html.

13. C. C. Stark and M. J. Kuchner, "A new algorithm for self-consistent three-dimensional modeling of collisions in dusty debris disks," Astrophys. J. 707(1), 543-553 (2009).

14. A. Roberge et al., "Finding the needles in the haystacks: high-fidelity models of the modern and Archean solar system for simulating exoplanet observations," Publ. Astron. Soc. Pac. 129, 124401 (2017).

15. H. Isaacson and F. D. Fischer, "Empirical limits on radial velocity planet detection for young stars," Astrophys. J. 725, 875 (2010).

16. B. J. Fulton et al., "RadVel: the radial velocity modeling toolkit," Publ. Astron. Soc. Pac. 130, 044504 (2018).

17. R. Soummer, L. Pueyo, and J. Larkin, "Detection and characterization of exoplanets and disks using projections on Karhunen-Loève Eigenimages," Astrophys. J. Lett. 755(2), L28 (2012).

18. S. Blunt et al., "orbitize!: a comprehensive orbit-fitting software package for the highcontrast imaging community," Astron. J. 159, 89 (2019).

19. S. Ulmer-Moll et al., "Beyond the exoplanet mass-radius relation," Astron. Astrophys. 630, A135 (2019).

Margaret C. Turnbull is an astronomer at the SETI Institute, specializing in nearby star systems, their habitable zones, and the technology to detect Earth-sized planets therein. She is a principal investigator for the Roman CGI Science Investigation Team.

Neil Zimmerman is a research astrophysicist in the Exoplanets and Stellar Astrophysics Laboratory at NASA's Goddard Space Flight Center. He is a member of the Nancy Grace 
Roman Space Telescope Project Science team, and his research encompasses coronagraph design and simulation, data processing techniques, and spectroscopy technologies for future exoplanet imaging missions.

Julien H. Girard is a scientist at the Space Telescope Science Institute. He serves as a lead coordinator for the Roman Exoplanet Imaging Data Challenge.

Sergi R. Hildebrandt is a scientist at the Jet Propulsion Laboratory and lecturer at California Institute of Technology. He received his $\mathrm{PhD}$ in theoretical physics from the University of Barcelona. He has worked in the data analysis of the cosmic microwave background from space and ground missions, adaptive optics in the visible, the Nancy Grace Roman Space Telescope, and more recently on leading the development of SISTER, a user friendly, open-source project that generates starshade simulations with high fidelity.

Zhexing $\mathbf{~ L i}$ is a $\mathrm{PhD}$ candidate at the University of California, Riverside, pursuing a degree in Earth and planetary sciences. His research interest is detecting and characterizing exoplanets through various detection methods and dynamical analysis. Currently, he is working on developing synergy between radial velocity and direct imaging campaigns.

Ell Bogat: Biography is not available.

Junellie Gonzalez Quiles is a PhD student in Earth and planetary sciences at Johns Hopkins University, where she works on connecting research in planetary interiors with currently available atmospheric data of known exoplanets, to fully understand the nature of such planets.

Christopher Stark is currently a deputy integration, test, and commissioning project scientist for JWST and the Large Strategic Mission Scientist at NASA Goddard Space Flight Center. His research focuses on future mission planning, exoplanets, and debris disks. He participated in the LUVOIR and HabEx Science and Technology Study Teams, served on the ExoPAG Executive Committee, and served on the Astro2020 decadal science panel on Exoplanets, Astrobiology, and the Solar System.

Avi Mandell is a civil servant research scientist in the Planetary Systems Laboratory (Code 693) at the NASA Goddard Space Flight Center. My research focuses on the formation and evolution of planetary systems, with the specific goal of understanding the factors that determine whether a system can form habitable planets and what the characteristics of these planets will be.

Tiffany Meshkat is an assistant research scientist at IPAC/Caltech where she studies the direct imaging of extrasolar planets. Her astronomical research focuses on understanding giant exoplanet formation through high contrast imaging. As a member of the WFIRST coronagraph instrument team, she contributes to the data processing pipeline and community proposal tools.

Stephen R. Kane is a professor of planetary astrophysics at the University of California, Riverside, with interests in exoplanet detection and characterization, planetary habitability, astrobiology, orbital dynamics, and studying Venus as an exoplanet analog. 